\title{
Social and Spatial Distance in Decision- Making: Can Culture Play a Role? An Abstract
}

\author{
Shuang Wu
}

\begin{abstract}
With the innovation of technology and growing usage of social media, integrated marketing communications are targeting the customers from worldwide. Construal level theory has been extensively used to explain the effect of psychological distance on evaluation, prediction, and behavior (Trope et al., 2007). Psychological distance is defined as "the subjective distance between an actor and an event in the actor's psychological space (Kim et al., 2008)." This multidimensional construct includes temporal distance, social distance, spatial distance, and hypotheticality (Liberman et al., 2007). Social distance and spatial distance are two dimensions that have been studied independently in previous literature. The author hypothesizes that the interaction effect of social distance and spatial distance on consumers' evaluation varies from Western culture to Eastern culture. Moreover, this study intends to offer insights into the use of construal level theory in tourism advertising. Destinations targeting visitors from different cultures might adjust their marketing communications to be appealing. In conjunction with the tourism context, this research reviews the effects of two psychological distance dimensions, social distance and spatial distance, and the potential moderating effect of culture on customers' attitudes and behavior intention. A $2 \times 2 \times 2$ experimental design is purposed to test the hypotheses.
\end{abstract}

References Available Upon Request

\footnotetext{
S. Wu $(\bowtie)$

Louisiana Tech University, Ruston, LA, USA

e-mail: swu007@latech.edu
} 